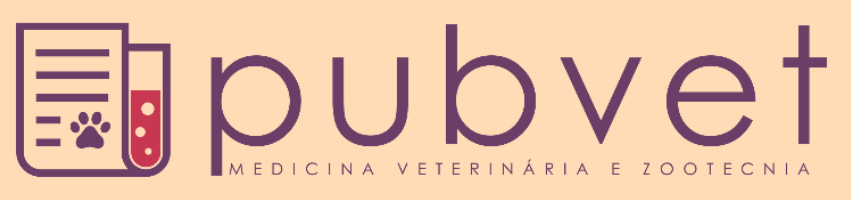

https://doi.org/10.31533/pubvet.v15n08a890.1-7

\title{
Perspectiva de tutores de cães e gatos de Manaus/AM sobre cardiopatias em pequenos animais
}

\author{
Talita Aparecida Mariano Fernandes Mello ${ }^{1 * \bullet}$, Marina Pandolphi Brolio ${ }^{2} \bullet$ \\ ${ }^{1}$ Acadêmica do curso de Medicina Veterinária, Centro Universitário Fametro - CEUNI FAMETRO. Manaus - AM Brasil. \\ ${ }^{2}$ Docente do curso de Medicina Veterinária, Centro Universitário Fametro - CEUNI FAMETRO. Manaus - AM Brasil. \\ *Autor para correspondência, E-mail: talita.mello@outlook.com
}

Resumo. As cardiopatias na clínica de pequenos animais estão entre as principais casuísticas em animais senis, isso se deve principalmente a nova consciência presente no trato dos tutores acerca do bem-estar animal, fator que implica no aumento recorrente das taxas de longevidade dos animais de companhia. Porém, nos termos da Medicina Preventiva Veterinária, observa-se relapsidade dos proprietários sobre a importância das consultas de rotina para a saúde geral dos cães e gatos, neste caso, direcionadas ao diagnóstico precoce e tratamento adaptado a promoção de uma melhor qualidade de vida para animais cardiopatas. Nesse contexto, o presente estudo investigou o conhecimento prévio da população de Manaus/AM sobre cardiopatias em pequenos animais e sua importância na rotina clínica veterinária para a aplicação da Medicina Veterinária Preventiva. Foram analisados dados obtidos em um questionário de cadastro respondido por 102 tutores de pets interessados em agendar atendimento aos animais na ação social "III Setembro Vermelho Animal", ocorrida nos dias 29 e 30 de setembro de 2020. O prérequisito para participação era o animal ter acima de cinco anos e o tutor responder todo o questionário através de um formulário online, aplicado entre 22 e 28 de setembro de 2020 , divulgado através de publicações em portais de noticiais e das redes sociais da coordenação e comissão organizadora do evento do curso de Graduação em Medicina Veterinária do CEUNI - FAMETRO, Manaus - AM.

Palavras-chave: Medicina preventiva veterinária, doença cardíaca, animais de companhia

\section{Perspective of dog and cat tutors from Manaus/AM about heart disease in small animals}

Abstract. Heart diseases in the small animal clinic are among the main casuistry in senile
animals, this is mainly due to the new awareness present in the tutors' treatment of animal
welfare, a factor that implies the recurrent increase in the longevity rates of companion
animals. However, under the terms of Veterinary Preventive Medicine, there is a relapsed
nature of the owners regarding the importance of routine consultations for the general
health of dogs and cats, in this case, aimed at early diagnosis and treatment adapted to the
promotion of a better quality of life for cardiopathic animals. In this context, the present
study investigated the previous knowledge of the population of Manaus / AM about heart
disease in small animals and its importance in the veterinary clinical routine for the
application of Preventive Veterinary Medicine. Data obtained from a registration
questionnaire answered by 102 pet tutors interested in scheduling care for animals in the
social action "III Setembro Vermelho Animal", which took place on September 29 and 30 ,
2020 , were analyzed. The prerequisite for participation was the animal is over five years
old and the tutor answers the entire questionnaire through an online form, applied between
22 and 28 September 2020, disseminated through publications on news portals and social 
networks of the coordination and organizing committee of the course event Graduation Course in Veterinary Medicine at CEUNI - FAMETRO, Manaus.

Keywords: Veterinary preventive medicine, heart disease, company animals

\section{Introdução}

Atualmente cães e gatos vivem mais do que décadas atrás devido aos avanços da medicina veterinária e ao desenvolvimento e oferta de fármacos de uso veterinário pela indústria farmacêutica. Soma-se a isto o crescimento do ramo pet, que tem posto à disposição dos proprietários de animais de estimação uma imensa gama de produtos, a qual despertou o interesse dos tutores sobre as questões relacionadas ao cão e gato geriátricos, bem como as dos profissionais de medicina veterinária de pequenos animais (Goldston \& Hoskins, 1999; Hoskins, 2008; Panegossi et al., 2017).

A realização do exame físico geral ou de rotina é necessária por inúmeras razões, entre as quais se destaca a incapacidade de comunicação e expressão verbal dos animais, conduzindo a uma dependência de conhecimento por parte do proprietário (Feitosa, 2014; Houston \& Radostits, 2002). Deste modo, como é regra geral, a prevenção dos problemas perla prática da medicina preventiva é, cada vez mais, o caminho mais eficaz a seguir em medicina veterinária (Pfuetzenreiter et al., 2004).

Castro et al. (2009) citam que o conhecimento da prevalência das afecções cardíacas em cães é de fundamental importância para o clínico de pequenos animais, pois auxilia na formulação de diagnósticos diferenciais e no estabelecimento de um plano terapêutico adequado. Visto que os animais de companhia têm apresentado queixas clínicas de patologias das quais não se discutiam há anos, em decorrência da modernização da medicina veterinária e do aumento de expectativa de vida desses, a investigação do conhecimento da população acerca das doenças cardíacas, da importância das consultas preventivas e a posterior orientação e esclarecimentos a esse público se tornam imprescindíveis.

O presente trabalho tem por objetivo analisar a percepção de tutores de Manaus, Amazonas sobre a ocorrência de cardiopatias e da importância da aplicação da medicina preventiva Veterinária em cães e gatos através de consultas de rotina clínica.

\section{Material e métodos}

A partir de embasamento teórico foi elaborado um formulário online, através da plataforma do Google Forms, com 46 questões de múltipla escolha e de característica subjetiva, relacionadas ao perfil socioeconômico dos tutores e características gerais dos animais participantes, além de perguntas sobre cardiopatias em cães e gatos e a prevalência de atendimentos de rotina ofertados a esses animais.

O formulário foi dividido em seções, a partir da confecção disponibilizada pela ferramenta do Google, sendo essas: informações do animal e tutor e histórico do animal. Dentre as questões presentes na seção histórico do animal, quatro eram direcionadas para os aspectos cardíacos da pesquisa. $\mathrm{O}$ formulário foi utilizado para triagem dos animais atendidos na Campanha "III Setembro Vermelho Animal" e ficou disponível para preenchimento entre os dias 22 e 28 de setembro de 2020. O linkpara o acesso ao questionário foi enviado por e-mail aos tutores participantes da campanha.

A ação social "III Setembro Vermelho Animal" foi aberta ao público e divulgada por canais digitais como as redes sociais, (Facebook e Instagram), do curso de Medicina Veterinária e do CEUNI FAMETRO. A campanha foi divulgada em diversos portais de notícias, dentre eles o site da Instituição e os portais online dos jornais Diário do Amazonas, Manaus Alerta, Rede Notícias e o jornal A Crítica. Também ocorreu o anúncio da ação através de listas de transmissão em aplicativos de mensagens (Whattsapp e Telegram), por intermédio dos acadêmicos e demais indivíduos envolvidos na coordenação do evento.

Na apresentação do formulário havia o Termo de Consentimento Livre e Esclarecido - TCLE, no qual estava especificada a permissão dos participantes para o uso das informações que seriam computadas, assim como a segurança e não divulgação de seus dados pessoais.

Com os dados obtidos pela aplicação do formulário foi realizada a tabulação das informações e posteriormente a elaboração de gráficos para ilustrar a porcentagem das questões de múltipla escolha 
assim como os índices comuns das questões subjetivas. Tendo em vista o caráter generalista do questionário aplicado, a fim de caracterizar a promoção do agendamento na participação da ação do "III Setembro Vermelho Animal", cinco questões foram selecionadas para gerar a discussão acerca do tema da presente pesquisa.

\section{Resultados e discussão}

Foram obtidos e analisados 102 questionários, com respostas de tutores de cães e gatos, pessoas de ambos os sexos, residentes no município de Manaus, Amazonas. Quanto ao perfil dos animais participantes da ação social; o pré-requisito foi ter a partir de cinco anos de idade, independente da espécie, raça e sexo e status sexual - se castrados ou não.

Dentre as questões selecionadas para discussão, primeiramente foi solicitado que os proprietários respondessem com que frequência levam seus pets a consultas veterinárias. A maioria dos tutores, 59,8\% do total de entrevistados (61 pessoas), informou o hábito de procurar atendimento médico veterinário somente em situações extremas, de doença aparente ou casos de emergência, enquanto 13,7\% afirmaram que nunca levaram seu pet ao veterinário, caracterizando uma parcela preocupante quando se trata de animais de companhia em geral, e nesse caso em específico em relação aos animais senis o quadro observado apresenta mais um agravante. Apenas 4,9\% dos participantes, relataram levar seus pets para consultas clínicas de rotina de forma regular (Gráfico 1).

Qual a frequência em que você leva seu PET ao veterinário?

102 respostas

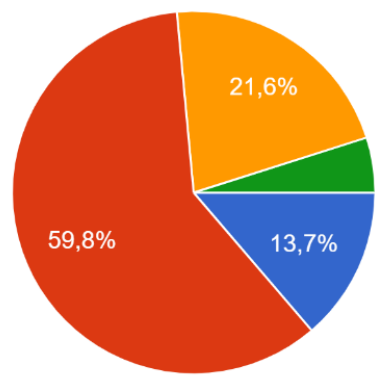

Nunca foi

Somente quando fica muito doente

Uma vez por ano para as vacinas

Regularmente, faz consultas de rotina

Gráfico 1. Frequência de atendimento veterinário.

Tais tabulações demonstram a falha comunicação entre o preceito de medicina veterinária Preventiva e a promoção da saúde em pequenos animais, a partir do posicionamento dos tutores a respeito da prioridade em levarem seus pets para consultas rotineiras ser secundária ao fator de emergência ou urgência expressa através somente da observação de uma sintomatologia já aparente (AAHA, 2011).

Dados recentes indicam que o maior motivo para tal problema é a falta de recomendação clara dos médicos-veterinários aos proprietários para a realização de exames completos regulares aos seus animais (Vogt et al., 2010).

Os dados obtidos pelas discussões promovidas por esse trabalho são reafirmados a partir da análise do estudo de Panegossi et al. (2017), no qual, dentre os tutores entrevistados que não visitaram o veterinário no último ano, uma parcela de 22 pessoas, dentre uma amostra de 30 tutores, representando $73,3 \%$ afirmou não ter procurado atendimento profissional, pois seu animal não apresentou nenhuma doença aparente. A autora inclusive relata que esse número só não foi superior devido ao ambiente de amostragem e abordagem desses entrevistados serem justamente em recepções de clínicas veterinárias.

Em concordância e de forma comparativa a interpretação do Gráfico 1, no Gráfico 2 problematizase ainda mais a compreensão de tutores sobre a importância do acompanhamento de rotina para animais de companhia. Pois, apesar de, em sua maioria, os tutores demonstrarem a baixa frequência em consultas clínicas veterinárias, vários animais participantes deste trabalho já foram diagnosticados com patologias relevantes, assim como foi afirmado por seus tutores, representando $72,5 \%$ do total de respostas. 
Gaspar (2018) disserta que de fato essa relapsidade dos tutores se trata de falta de conhecimento, que as observações de agravamento dos sinais clínicos apresentados por um animal só pode ser realmente identificado por um tutor se esse possuir "um olhar treinado", ou seja noções básicas em saúde seja por formação ou busca por informações (inclusive online), possibilitando a observação de eventuais alterações no corpo do pet, o que o incapacita de realizar um diagnóstico de patologia relevante, ou seja, a percepção do que seria uma doença de protocolo simples ou complexo tratamento.

Em sequência foi solicitado que os participantes relatassem se o seu animal de estimação já havia feito alguma avaliação cardiológica com um médico veterinário. Noventa e dois porcento dos tutores da amostra responderam negativamente. A partir desse resultado, apresentado no Gráfico 3, ressalta-se novamente a negativa em relação a percepção dos tutores acerca de patologias que são agravantes à idade avançada dos animais. Slupe et al. (2008) descrevem que as patologias cardíacas são uma das principais causas de morte em cães, portanto assim como a aplicação da medicina preventiva veterinária generalista é fundamental, as especialidades, tal como as cardiopatias, requerem um acompanhamento estreito de rotina.

Seu animal já foi diagnosticado com alguma doença relevante? 102 respostas

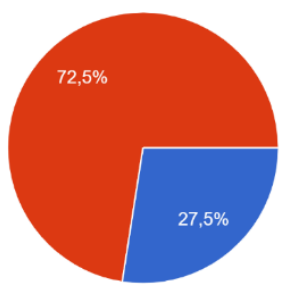

Gráfico 2. Diagnóstico de patologia prévia.
Seu animal já passou por avaliação cardiológica com veterinário?
102 respostas

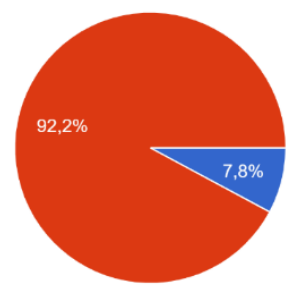

nim

Tilley \& Smith (2015) descrevem a importância da investigação clínica ao dizer que o diagnóstico precoce das diferentes doenças que afetam o sistema cardiovascular em cães e gatos é fundamental, para estabelecer o prognóstico e o tratamento adequado para cada paciente. Estudos epidemiológicos estimam uma incidência na população canina que varia entre $17 \%$ e $40 \%$, já, outros autores estimam um maior percentual de até 58\% em cães com mais de nove anos e até mesmo 75\% em animais de mais 16 anos de idade (Ettinger et al., 2002, 2017; Hoskins, 2008).

A respeito da realização de exames direcionados a cardiologia veterinária, a maioria dos tutores expressou que seus animais de companhia nunca realizaram nenhum dos procedimentos relatados nessa questão, correspondendo a $88,2 \%$ do total da pesquisa. Dentre os demais dados fornecidos pelos entrevistados, 5,9\% informaram que seu pet já realizou aferição da pressão arterial e 4,9\% que seu animal de companhia já fez um ecocardiograma (Gráfico 4).

Seu animal já fez algum desses exames?

102 respostas

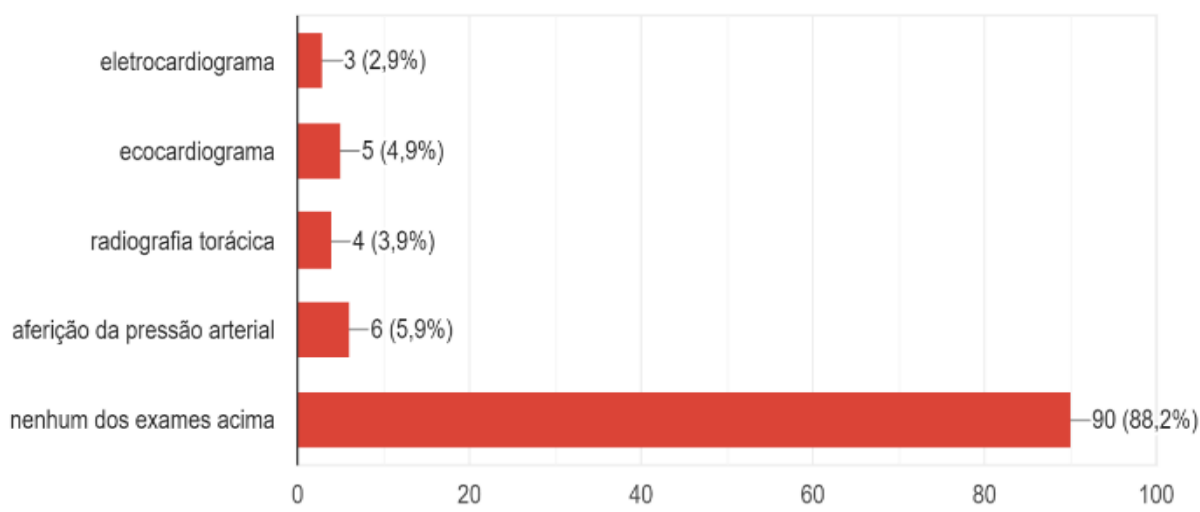

Gráfico 4. Realização de exames cardiológicos. 
O resultado encontrado na presente enquete é preocupante, pois com o aumento da expectativa de vida de cães e gatos, e sabendo-se que o sucesso de um tratamento está relacionado com a detecção precoce de algumas doenças, aumenta a busca por técnicas diagnósticas complementares seletivas e precisas que auxiliem no diagnóstico das patologias, para contribuir com tratamentos eficazes e acima de tudo, que ofereçam qualidade de vida aos animais (Brown \& Henik, 2002).

A apresentação de poucos animais com controle e manejo preventivo de cardiopatias referencia às notificações de desaviso acerca de medicina preventiva; porém, além disso, ressignifica as próprias abordagens de médicos veterinários no tocante a cirurgias eletivas, se observado que alguns cães e gatos da pesquisa foram declarados como castrados pelos proprietários, todavia uma minoria esmagadora nunca realizou se quer um eletrocardiograma, por exemplo. Entretanto, alguns estudos demonstram que a avaliação eletrocardiográfica pré-cirúrgica nos pacientes é de grande relevância, incluindo-se os casos em que o exame físico não indica doença cardíaca, pois as alterações observadas não dependem da manifestação de sinais clínicos de cardiopatia. Além disso, alguns autores ressaltam que a presença de alterações eletrocardiográficas é independente da faixa etária do animal, sendo essencial que o exame seja realizado inclusive em cães jovens (Carvalho et al., 2009). O envolvimento de um processo crônico normalmente está relacionado a fatores de risco próprios da idade (Jericó et al., 2015), alertando sobre a necessidade de submeter estes pacientes a exames cardiológicos de rotina (Lima \& Costa, 2015).

$\mathrm{Na}$ última questão averiguada foi solicitado que o tutor relatasse se o animal que seria atendido na ação social do "III Setembro Vermelho Animal" apresentava algum dos sintomas descritos na questão, sendo estes compatíveis com um possível diagnóstico de cardiopatia. Como é constatado no Gráfico 5, na tabulação, metade dos entrevistados, 51 pessoas, afirmaram que os seus animais de companhia apresentavam fadiga e cansaço fácil, enquanto 35 tutores responderam que seus pets possuíam respiração acelerada.

Esses dados descritos no Gráfico 5, corroboraram com as afirmações de Vincenzi (2009), visto que o autor explana que os sinais clínicos mais comumente encontrados na doença cardíaca são apatia, intolerância ao exercício físico, redução de apetite, diminuição do peso corporal, tosse, dificuldade respiratória, cianose, síncope, distúrbios no crescimento em filhotes e nas atividades físicas de cães atletas.

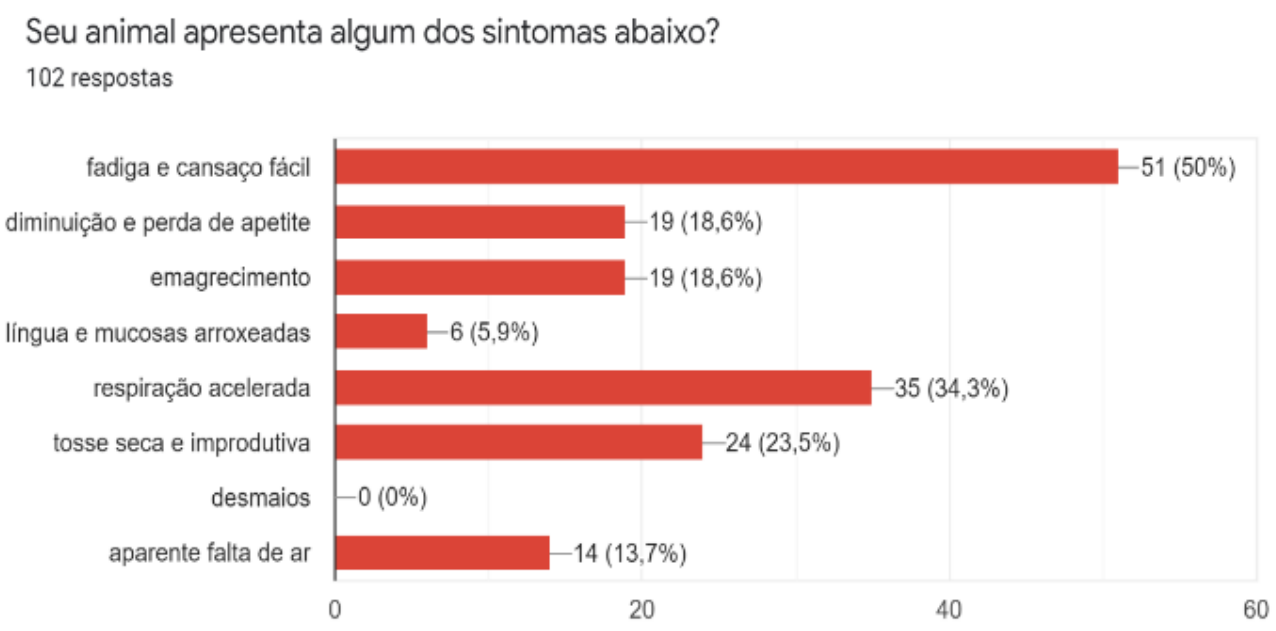

Gráfico 5. Apresentação de sintomatologia indicativa de cardiopatia.

As doenças associadas ao sistema cardiovascular apresentam evolução silenciosa, caracterizada pela fase atenuante ser descrita como o princípio de aparecimento dos sinais clínicos em si. A sinalização de algumas anormalidades pelos pacientes cardiopatas pode, por anos, passar despercebida à observação dos proprietários, propiciando a evolução dos sinais clínicos (Belerenian et al., 2003; Ettinger et al., 2002, 2017). Essa progressão sintomatologia silenciosa a sutis modificações de comportamento até o 
aparecimento em si de piora de quadro clínico pode ser justificada pela falta de observação dos tutores e da consequente surpresa na descoberta de um diagnóstico de cardiopatia.

A análise dos resultados apontam falha na rotina de realização de exames e consultas clínicas voltadas para avaliação cardiológica, correlacionada à imprecisão de informações e discernimento dos participantes frente a ocorrência de cardiopatias em cães e gatos, bem como a importância da prevenção cardiológica e o aumento do índice de mortalidade de cães e gatos por cardiopatias, visto que cerca de $11 \%$ dos animais de companhia possuem alguma doença cardiovascular, sendo essa uma causa comum de morbidade e mortalidade em cães idosos.

Tendo em vista que a sintomatologia apresentada aos entrevistados, através das alternativas disponibilizadas pelo formulário e visíveis no Gráfico 5, é somente indicativa de diagnóstico, ou seja, não possui nenhum caráter patognomônico de patologia cardiológica, pode-se apontar não somente o quesito abordado da atitude relapsa dos tutores frente ao desenvolvimento de sinais clínicos aparentes e agravantes ao bem estar do animal de estimação desses, como discutido anteriormente, mas também o enfoque desenfreado da falta de acompanhamento de animais em consultas de rotina para a identificação prévia de qualquer doença.

Nelson \& Couto (2015) evidencia a importância do uso de meios auxiliares de diagnóstico, para que possa ser estabelecida tanto uma identificação precoce quanto um tratamento preciso de cardiopatias em animais com idade avançada, visto que, muitas vezes, esses pacientes ainda não apresentam sinais clínicos específicos dessas doenças e já apresentam diversas alterações nos exames realizados.

\section{Conclusão}

O presente trabalho avaliou o conhecimento prévio dos tutores de cães e gatos sobre cardiopatias em animais de companhia e a importância da Medicina Preventiva Veterinária para o bem-estar animal. Apesar das cardiopatias terem índices crescentes de incidência e mortalidade de cães e gatos, observouse nessa pesquisa que os proprietários, em sua maioria, não têm o hábito de submeter seus animais a nenhum tipo de consulta especializada ou exame complementar referente a investigação cardiológica. Tal dado se alia com outra informação fornecida, a de que alguns animais da amostragem possuem sintomatologia compatível com alguma cardiopatia, de modo a salientar a falta de conhecimento dos proprietários a respeito da observação de um quadro sintomatológico correspondente a um caso de cronicidade, ou seja, de uma piora do estado de saúde do animal inviabilizando um tratamento precoce.

O caráter focalizado de emergência e urgência quando se trata de atendimentos veterinários se torna claro quando os entrevistados afirmam que apenas levam seus animais para consulta quando esses se encontram muito doentes ou em situações de reforços vacinais. Se tratando de tratamento precoce, de maneira geral, se nota necessidade da propagação da medicina preventiva em si, e por conta do desconhecimento dos tutores, comprovado através das análises das questões respondidas por esses, cabem aos médicos veterinários o papel de difundir a importância das consultas de acompanhamento aos animais de companhia. A senilidade, de forma geral, representa uma fase de sensibilização do organismo, o que requer atenção dobrada para a manutenção em saúde dos animais, tendo em vista que, além de estarem cada vez mais presentes nos núcleos familiares, os pets ainda possuem a desvantagem de não poderem se comunicar em uma mesma linguagem conosco.

As ações sociais são divisoras de água na comunidade, por se responsabilizarem em manter um contato direto com a população alvo, possibilitam a conscientização sobre diversos temas importantes, como cardiopatias em cães e gatos e a medicina veterinária preventiva, além de fornecer atendimentos e serviços básicos aos participantes. No que se diz respeito aos alunos envolvidos, a experiência acadêmica de lidar, antes da atuação como profissional propriamente dita, com o público fornece humanização do atendimento ao animal e a capacidade de estudar e aplicar saberes de especialidades veterinárias.

\section{Referências}

AAHA (2011). American Animal Hospital Association. Development of New Canine and Feline Preventive Healthcare Guidelines Designed to Improve Pet Health. Journal of the American Hospital Association, v. 47, 306-311. 
Belerenian, G. C., Mucha, C. J., \& Camacho, A. A. (2003). Afecções cardiovasculares em pequenos animais. Interbook.

Brown, S. A., \& Henik, R. A. (2002). Hipertensão sistêmica. In L. P. Tilley, J.-K. Goodwin, J. J. Fagliari, G. S. Fagliari, M. T. Costa, J. N. B. Andrade, M. L. Rezende, \& N. N. Silva (Eds.), Manual de cardiologia para cães e gatos. Roca.

Carvalho, C. F., Tudury, E. A., Neves, I. V, Fernandes, T. H. T., Gonçalves, L. P., \& Salvador, R. (2009). Eletrocardiografia pré-operatória em 474 cães. Arquivo Brasileiro de Medicina Veterinária e Zootecnia, 61(3), 590-597. https://doi.org/10.1590/s0102-09352009000300011.

Castro, M. G., Veado, J. C. C., Silva, E. F., \& Araújo, R. B. (2009). Estudo retrospectivo ecodopplercardiográfico das principais cardiopatias diagnosticadas em cães. Arquivo Brasileiro de Medicina Veterinária e Zootecnia, 61(5), 1238-1241. https://doi.org/10.1590/S010209352009000500032.

Ettinger, S. J., Fedlman, E. C., \& Taibo, R. A. (2002). Tratado de medicina interna veterinaria: enfermedades del perro y el gato. Manole.

Ettinger, S. J., Feldman, E. C., \& Cote, E. (2017). Textbook of Veterinary Internal Medicine-eBook. Elsevier Health Sciences.

Feitosa, F. L. F. (2014). Semiologia veterinária: A arte do diagnóstico. Grupo Gen-Editora Roca Ltda.

Gaspar, S.S (2018). Cães e gatos como pacientes: uma etnografia de um hospital veterinário. Tese de doutorado apresentada ao Programa de PósGraduação em Antropologia Social da Universidade Federal do Rio de Janeiro: Rio de Janeiro - RJ.

Goldston, R. T., \& Hoskins, J. D. (1999). Geriatria e gerontologia do cão e do gato. Rocca.

Hoskins, J. D. (2008). Geriatria e Gerontologia do cão e do gato. Editora Roca.

Houston, D. M., \& Radostits, O. M. (2002). O exame clínico. Koogan Guanabara.

Jericó, M. M., Kogika, M. M., \& Andrade Neto, J. P. (2015). Tratado de medicina interna de cães e gatos. Guanabara Koogan.

Lima, M. M. C., \& Costa, P. P. C. (2015). A importância da avaliação ecocardiográfica em cães com neoplasia tratados com doxorrubicina. Relato de caso. Revista Brasileira de Higiene e Sanidade Animal, 9(3), 518-527.

Nelson, R. W., \& Couto, C. G. (2015). Medicina interna de pequenos animais (Issue 1). Elsevier Editora.

Panegossi, M. F. C., Marques, A. E. G. W., L., S. N., Marques, M. G., Nagata, W. B., \& Bresciani, K. D. S. (2017). Necessidade de orientação dos tutores sobre posse responsável canina. Ars Veterinaria, 32(2), 88-91. https://doi.org/10.15361/2175-0106.2016v32n2p88-91.

Pfuetzenreiter, M. R., Zylbersztajn, A., \& Avila-Pires, F. D. de. (2004). Evolução histórica da medicina veterinária preventiva e saúde pública. Ciência Rural, 34(5), 1661-1668. https://doi.org/10.1590/s0103-84782004000500055.

Slupe, J. L., Freeman, L. M., \& Rush, J. E. (2008). Association of body weight and body condition with survival in dogs with heart failure. Journal of Veterinary Internal Medicine, 22(3), 561-565. https://doi.org/10.1111/j.1939-1676.2008.0071.x.

Tilley, P. L., \& Smith, F. K. W. (2015). Five-minute Veterinary consult: canine and feline. John Wiley $\&$ Sons.

Vincenzi, M (2009). Atualizações sobre o uso de óleo de peixe no tratamento de cardiopatia canina. Trabalho de Conclusão de Curso de Graduação em Medicina Veterinária. Unesp: Botucatu - SP.

Vogt, A. H., Rodan, I., Brown, M., Brown, S., Buffington, C. A. T., Forman, M. J. L., Neilson, J., \& Sparkes, A. (2010). AAFP-AAHA: feline life stage guidelines. Jornal of Feline Medicine and Surgery, 12, 43-54.

Histórico do artigo:

Recebido: 8 de maio de 2021

Aprovado: 31 de maio de 2021
Licenciamento: Este artigo é publicado na modalidade Acesso Aberto sob a licença Creative Commons Atribuição 4.0 (CC-BY 4.0), a qual permite uso irrestrito, distribuição, reprodução em qualquer meio, desde que o autor e a fonte sejam devidamente creditados. 